\title{
Medication Adherence in patients with systemic lupus erythematosus: A descriptive study
} Sofia Jami Alahmadi ${ }^{1}$, Seyedeh Tahereh Faezi², Mahdi Mahmoudi², Leila Sayadi ${ }^{3 *}$

${ }^{1}$ School of Nursing \& Midwifery, Tehran University of Medical Sciences, Tehran, Iran. ${ }^{2}$ Rheumatology Research Center, Tehran University of Medical Sciences, Tehran, Iran. ${ }^{3}$ Nursing and Midwifery Care Research Center, School of Nursing \& Midwifery, Tehran University of Medical Sciences, Tehran, Iran.

Systemic lupus erythematosus is an autoimmune disease that affects several systems of the body. Due to the chronic nature of this disease, non-adherence to a medication regimen can lead to deterioration by the disease and also misinterpretation of treatment results. The aim of this study was to determine the medication adherence of patients with systemic lupus erythematosus.

In this descriptive study, medication adherence was investigated in 209 patients with SLE who were attending a lupus clinic in one of the hospitals affiliated with Tehran University of Medical Sciences. The sample participants who fit inclusion criteria were entered in the study by the convenience and continuous method. Then, they completed a demographic information form and the eight-item Morisky medication adherence scale. Data were analyzed by SPSS software using descriptive statistics, chi-square, and the ANOVA test.

The results showed that $18.7 \%$ of patients had high adherence to medication regimen, 94 patients $(45.0 \%)$ had low adherence, and 76 patients $(36.4 \%)$ had moderate level of adherence to medication. There was no significant relationship between the demographic and clinical variables of patients and their adherence to their medication.

It is necessary to take measures to increase medication adherence among these patients with systemic lupus erythematosus due to their low and moderate medication adherence. It is also necessary that nurses and physicians in the clinic provide patient education and heed this issue in medical and nursing programs.

Keywords: Systemic lupus erythematosus, Medications adherence, Non-adherence to medication

\section{Introduction}

Systemic lupus erythematosus (SLE) is a multisystem autoimmune disease of a chronic nature, which has periods of recurrence and remission [1]. In this disease, antibodies are produced that act against the body cells instead of bacteria, which results in the production of immune complexes that destroy body cells by circulating in the bloodstream and depositing in various tissues [2]. Involvement of different body systems causes many problems and disabilities for patients, leading to dependence and difficulty in meeting health care needs. These issues not only have high costs for patients [3], but also impose significant costs on the health care system, especially when disease severity elevates [4].

Although the treatment of SLE varies depending on its stage, it must be followed for the remaining life of the patient due to its chronic nature. Pain management in these patients by non-steroidal anti-inflammatory medications and weakening of the immune system by immunosuppressive medications are the most important therapeutic goals in SLE. Immunosuppressive medications such as tacrolimus, mycophenolate, corticosteroids, cyclophosphamide, azathioprine, methotrexate, as well as medications that inhibit the production of B-lymphocytes (blimombe) and chloroquine, are among the medications used in this disease [5]. Adherence of patients to their medication regimen is very important in the success of treatment and improvement of these patients, and it is one of the important components of self-management in SLE [6]. However, studies have shown that in diseases of a chronic nature, patients do not adhere to medication and avoid taking medications or change the dose of medications spontaneously. Non-adherence to medication regimen hinders the effective management of the disease and leads to negative health outcomes, worsening of the disease, hospitalization, and even death among SLE

Personal non-commercial use only. Rheumatology Research Journal. Copyright $($ 2020. All rights reserved

*Corresponding Author: Leila Sayadi, Nursing and Midwifery Care Research Center, School of Nursing \& Midwifery, Tehran University of Medical Sciences, Tehran, Iran. E-mail: 1-sayadi@sina.tums.ac.ir, Telefax: 0098 (21) 61054325, 0098 (21) 66904252.

Received: 09 September 2019; Accepted: 10 October 2019 
patients $[7,8]$. Studies have reported $40 \%$ to $64 \%$ nonadherence to medication regimen among SLE patients [912], and non-adherence to medication in SLE patients has been reported as one of the important reasons for the worsening of their condition $[6,8]$.

Due to the importance of adherence to medication regimen, it is necessary to evaluate medication adherence of patients periodically, and depending on the result of this evaluation, implement appropriate interventions. This issue is particularly important in patients with SLE, because the periods of recurrence and remission of this disease are highly dependent on the adherence of these patients to the prescribed medications as it can decrease the disease activity $[10,13,14]$. Clinical studies cannot be interpreted realistically without having medication adherence information, and the effectiveness of treatment in studies in which patients show low levels of medication adherence is miscalculated, so patient medication adherence should be precisely considered in these clinical studies [8]. Although studies on non-adherence to medication regimen in SLE patients have been conducted in different countries, studies conducted in Iran on this issue are limited. This is while, in Iran, SLE has a prevalence of 40 per 100,000 and this number is increasing so that hospitals are facing multiple referrals and hospitalization of SLE patients. This issue is particularly important in Iran, because younger people in Iran are affected by this disease $[15,16]$. Furthermore, SLE patients in Iran are living with this disease for a longer period of their lives, so their medication adherence is very important for their treatment. This study, therefore, was performed to evaluate the medication adherence of patients with SLE in Iran.

\section{Materials and Methods}

This descriptive study was performed between May and August 2019.

\section{Setting \& participants}

This study was performed in one of the hospitals affiliated with Tehran University of Medical Sciences which is one of the main centers for referral and treatment of SLE in Iran. A total of 209 patients with SLE attending the SLE clinic were included in the study by the convenience and continuous method. Inclusion criteria for these patients included being over 18 years old, having ability to understand and speak Farsi, having the ability to read and write, giving consent to participate in the study, having a confirmed diagnosis of SLE by a specialist, and having a follow-up and treatment file in the clinic for at least one year. Any patient who refused to complete the questionnaire was excluded from the study, although this was not the case in this study.

\section{Data collection}

Data collection tools used in this study included a demographic and clinical information questionnaire and the eight-item Morisky medication adherence scale (MMAS-8). The MMAS-8 is a structured self-report scale and measures behaviors such as medication adherence. This scale facilitates the identification of behaviors associated with adherence to medications and related barriers. The MMAS- 8 scale contains 8 items; the first seven have a yes and no response and the last item is rated using the 5-point Likert's scale. In this study, yes and no were scored as zero and one, respectively, except for item 5 that was scored in reverse. The last item obtained the score of 1 (never) or 0 (once in a while, sometimes, usually, all the time). The sum of scores was ranged from 0 to 8 . The scores in this scale would fall in three levels of high or good adherence (score $=8$ ), medium adherence (score 6 to less than 8 ), and low or poor adherence (score less than 6) $[7,10]$

The MMAS- 8 has been translated into Farsi and has been used in various studies in Iran $[17,18]$. In this study, the MMAS-8 scale was given to ten nursing faculty members and rheumatologists and after being approved by them, its reliability was assessed. The reliability of medication adherence was calculated at $82 \%$ using Cronbach's alpha.

After obtaining informed consent from the participants, the data were collected using the above tools. The questionnaires were completed by the patients in a separate room in the clinic and, if necessary, instructions were given to them by the researcher.

\section{Statistical Analysis}

The SPSS software version 16 was used to analyze the data using frequency percentage, mean, and standard deviation as well as the chi-square and ANOVA tests.

\section{Results}

The mean age of the participants was 41.32 years with a standard deviation of 12.15 . Of the patients, $91.9 \%$ were female and the rest were male. The mean duration of illness in the patients was 137.8 months (Table 1).

Table 1. Demographic and clinical characteristics of the studied patients

\begin{tabular}{llc}
\hline & & N (\%)/ Mean \pm SD \\
\hline Age & & $41.32 \pm 12.15$ \\
\hline \multirow{2}{*}{ Gender } & Male & $17(8.1)$ \\
& Female & $192(91.9)$ \\
\hline
\end{tabular}




\begin{tabular}{llc}
\hline & & N (\%) / Mean \pm SD \\
\hline \multirow{2}{*}{ Marital state } & Single & $\mathbf{5 3}(\mathbf{2 5 . 4 )}$ \\
& Married & $\mathbf{1 5 6}(\mathbf{7 4 . 6 )}$ \\
\hline \multirow{2}{*}{ Education level } & Below diploma & $\mathbf{9 0}(\mathbf{4 3 . 1})$ \\
& Diploma & $\mathbf{7 3 ( 3 4 . 9 )}$ \\
& University & $\mathbf{4 6}(\mathbf{2 2})$ \\
\hline Duration of illness (month) & & $\mathbf{1 3 7 . 8} \pm \mathbf{1 1 1 . 4}$ \\
\hline Number of medications taken & & $\mathbf{5 . 1 7} \pm \mathbf{1 . 2 9}$ \\
\hline
\end{tabular}

Analysis of the participant responses showed that 96 patients $(45.93 \%)$ sometimes forgot to take their medications, and for 121 patients $(57.89 \%)$ daily use of medication was upsetting to them and they felt bad about following the treatment plan (Table 2).

Table 2. Frequency of drug adherence in the MMAS- 8 among patients under the study

\begin{tabular}{|c|c|c|c|}
\hline \multirow[t]{2}{*}{ No } & \multirow[t]{2}{*}{ Question } & \multicolumn{2}{|l|}{$\mathbf{N}(\%)$} \\
\hline & & Yes & No \\
\hline 1 & Do you sometimes forget to take your medication? & $96(45.93)$ & $113(54.07)$ \\
\hline 2 & $\begin{array}{l}\text { Sometimes people do not take their medicine because they forget } \\
\text { to do so. Have you been forgetting to take your medicine in the } \\
\text { last } 2 \text { weeks? }\end{array}$ & $63(30.14)$ & $146(69.86)$ \\
\hline 3 & $\begin{array}{l}\text { Have you ever stopped taking your medicine without consulting } \\
\text { your doctor because you felt unwell after taking it? }\end{array}$ & $39(18.66)$ & $170(81.34)$ \\
\hline 4 & $\begin{array}{l}\text { Have you ever forgotten to take your medicine while traveling or } \\
\text { leaving home? }\end{array}$ & $24(11.48)$ & $185(88.52)$ \\
\hline 5 & Did you take all your medicines yesterday? & $189(90.43)$ & $20(9.57)$ \\
\hline 6 & $\begin{array}{l}\text { Have you ever stopped taking your medication because you felt } \\
\text { that your symptoms were under control? }\end{array}$ & $21(10.05)$ & $188(89.95)$ \\
\hline 7 & $\begin{array}{l}\text { Taking daily medication is really upsetting for some people. Have } \\
\text { you ever felt the bad for following your treatment plan? }\end{array}$ & $121(57.89)$ & $88(42.11)$ \\
\hline 8 & How often do you forget to take your medication? & $\begin{array}{c}\text { once in a while/ sometimes/ } \\
\text { usually/ all the time } \\
109(52.2)\end{array}$ & $\begin{array}{l}\text { Never/rarely } \\
100(47.8)\end{array}$ \\
\hline
\end{tabular}

According to the results, 94 patients $(45.0 \%)$ had low adherence to medication, 76 patients $(36.4 \%)$ had moderate adherence, and 39 patients (18.7\%) had high adherence to the medication (Table 3).
There was no significant relationship between demographic and clinical variables of the patients and adherence to their medication (Table 4).

Table 3. Drug adherence classification of the patients

\begin{tabular}{lc}
\hline \multicolumn{1}{c}{ Drug adherence } & N (\%) \\
\hline High adherence & $39(18.7)$ \\
Moderate adherence & $76(36.4)$ \\
Low adherence & $94(45.0)$ \\
\hline
\end{tabular}


Table 4. Relationship between demographic and clinical variables, and drug adherence

\begin{tabular}{|c|c|c|c|c|c|}
\hline & & \multicolumn{3}{|c|}{ Drug adherence } & \multirow{2}{*}{$P$ value } \\
\hline & & high & Moderate & Low & \\
\hline \multicolumn{2}{|l|}{ Age* } & $41.97 \pm 12.56$ & $42.29 \pm 11.94$ & $40.27 \pm 12.20$ & 0.524 \\
\hline \multirow{2}{*}{ Sex! } & Male & $4(1.9)$ & $7(3.4)$ & $6(2.9)$ & \multirow{2}{*}{0.691} \\
\hline & Female & $35(16.7)$ & $69(33)$ & $88(42.1)$ & \\
\hline \multirow{3}{*}{ Education! } & Under diploma & $14(6.7)$ & 39 (18.7) & $37(17.7)$ & \multirow{3}{*}{0.412} \\
\hline & Diploma & $14(6.7)$ & $24(11.5)$ & $35(16.7)$ & \\
\hline & University & $11(5.3)$ & $13(6.2)$ & $22(10.5)$ & \\
\hline \multicolumn{2}{|c|}{ * Duration of illness (months) } & $137.08 \pm 95.98$ & $125.79 \pm 102.67$ & $147.81 \pm 123.64$ & 0.442 \\
\hline
\end{tabular}

\section{Discussion}

Findings of this study showed that the mean age of patients was 41.32 years with a standard deviation of 12.15 , and that the majority of patients were female. As indicated by numerous studies, SLE is also more common in women $[10,19,20]$. The mean age of patients in the studies conducted in different countries is close to the one in the present study. For example, the average age of patients was reported to be 35 years in a study conducted in 14 centers in Europe [20].

The results of the present study showed that $45.0 \%$ of patients had low medication adherence, $36.4 \%$ had moderate adherence, and $18.7 \%$ had high level of medication adherence. The results of a study conducted in Brazil, in which medication adherence of 55 patients with rheumatoid arthritis and 37 patients with SLE were assessed using Morisky scale, showed that medication adherence in patients with rheumatoid arthritis was $16.4 \%$ and in patients with SLE was 45.9\% [19]. Another study in Saudi Arabia, conducted on medication adherence of 140 patients with SLE using the Morisky Medication Adherence Scale, showed that $62.1 \%$ of patients did not adhere to medication regimen [10]. In a systematic review conducted in 2017 to investigate the lack of medication adherence in patients with SLE, the prevalence of nonadherence to medication regimen was $43 \%$ to $75 \%$ [21]. In 2019 in Indonesia, 36.6\% of patients (42 patients) with SLE did not adhere to medication regimen [22]. In another study conducted in Spain in 2018, the medication adherence of 72 women with SLE was measured and the results showed that $64 \%$ of patients did not adhere to the medication regimen [11]. However, results of a study conducted in Germany in 2018 on medication adherence of patients with SLE showed that $62.7 \%$ of patients had high adherence to medication regimen [23]. It should be noted that this issue is equally significant in other rheumatic diseases such as rheumatoid arthritis [24] as well as with other chronic diseases. For example, the results of a study conducted in Pakistan on antihypertensive medication adherence in patients with hypertension [25] showed that the prevalence of non-adherence among patients was high.
The findings also showed that reasons such as forgetting or feeling bad about taking medications were the most important cases of non-adherence to medication regimen. In other studies, it has been found that one of the reasons for medication non-adherence is patient forgetfulness in taking medication intentionally or unintentionally [8]. Other studies refer to concerns about the side effects of medications, believing in the harmfulness of medications, low perceptions and misconceptions about medications, multiple medications, and complex and lengthy treatment regimen as some of the reasons for lack of medication adherence $[6,8,10,22,23]$.

The results of studies show that medication adherence is a significant problem in patients with SLE and requires attention and intervention. Since ensuring patient adherence to medication therapy is the responsibility of a health care team, multidisciplinary approaches have been proposed to promote medication adherence in patients [19]. Therefore, it is essential that physicians and nurses teach medication adherence to patients in clinics or wards where SLE patients are being admitted. In this regard, a location in the clinic could be considered for performing this proposal and physicians or nurses could educate patients about this issue. Physicians and nurses should also take measures such as enhancing communication with patients and the treatment team, increasing patient motivation to take medication, and considering the individual characteristics of each patient in order to promote medication adherence [8]. We can find strategies to promote medication adherence and implement educational programs in hospitals to train nursing staff on this issue by conducting the pertinent research.

\section{Limitation}

It was not possible to measure medication adherence objectively by counting the medications taken or measuring the serum level of medications, so medication adherence of patients was measured using a questionnaire by a selfreporting method, which could have affected the accuracy of the results. Other factors including economic, disabilities, 
and disease severity may have affected the medication adherence that has not been reported in this study.

\section{Conclusion}

The results of the present study showed that a majority of patients had low or moderate levels of adherence to their medication. Therefore, it is necessary to take measures such as educating patients and medical staff, such as nurses who would have more contact with the patients, about the need for medication adherence and the consequences of non-adherence to a medication regimen. The issue of nonadherence to treatment and medication regimen could be added to the medical and nursing curriculum by developing effective strategies to reduce non-adherence to medication.

\section{Acknowledgments}

This study is part of a research project approved by the Institutional Research Ethics Committee, School of Nursing and Midwifery \& Rehabilitation - Tehran University of Medical Sciences, code: IR.TUMS.FNM.REC.1397.207. Study has been funded and supported by the Nursing and Midwifery Care Research Center, Tehran University of Medical sciences; Grant no. 97-03-99-40546. The authors of this study would like to thank those who helped us in this project.

\section{Conflict of interest}

The authors report that they have no conflicts of interest to declare.

\section{References}

1. Ferenkeh-Koroma A. Systemic lupus erythematosus: nurse and patient education. Nurs Stand 2012; 26(39):4957. doi: $10.7748 / \mathrm{ns} 2012.05 .26 .39 .49 . \mathrm{c} 9134$.

2. Yeoh S-A, Dias SS, Isenberg DA. Advances in systemic lupus erythematosus. Medicine 2018;46(2):84-92. doi.org/10.1016/j.mpmed.2017.11.010

3. AzadP FM. Experience of living with Systemic Lupus Erythematosus: A phenomenological study. Med Surg Nurs J 2015; 4(1):1-9.

4. Hammond E, Freidel H, Garal-Pantaler E, Pignot M, Velthuis E, Wang X. et al. PS8:160 Health care resource use (hru) and medical cost analyses as a function of systemic lupus erythematosus (sle) disease severity: analysis of claims data of a german sickness fund. Lupus Sci Med 2018; 5(Suppl 1): A116-A116. doi.org/10.1136/lupus-2018-abstract.203.

5. Fortuna G, Brennan MT. Systemic lupus erythematosus: epidemiology, pathophysiology, manifestations, and management. Dent Clin North Am 2013; 57(4):631-55. doi: 10.1016/j.cden.2013.06.003.

6. Lam WY, Fresco P. Medication adherence measures: an overview. Biomed Res Int 2051; 2015:217047. doi: $10.1155 / 2015 / 217047$

7. Tan X, Patel I, Chang J. Review of the four item Morisky medication adherence scale (MMAS-4) and eight item Morisky medication adherence scale (MMAS-8). Innov Pharmacy 2014; 5(3):5. doi.org/10.24926/iip.v5i3.347.

8. Ahmed I, Ahmad NS, Ali S, Ali S, George A, Saleem Danish H. et al. Medication Adherence Apps: Review and Content Analysis. JMIR Mhealth Uhealth 2018; 6(3):e62. doi: 10.2196/mhealth.6432.

9. Scalzi LV, Hollenbeak CS, Mascuilli E, Olsen N. Improvement of medication adherence in adolescents and young adults with SLE using web-based education with and without a social media intervention, a pilot study. Pediatr Rheumatol Online J 2018; 16(1):18. doi: 10.1186/s12969-018-0232-2

10. Alsowaida N, Alrasheed M, Mayet A, Alsuwaida A, Omair MA. Medication adherence, depression and disease activity among patients with systemic lupus erythematosus. Lupus 2018; 27(2):327-32. doi: $10.1177 / 0961203317725585$.

11. Prados-Moreno S, Sabio JM, Pérez-Mármol JM, Navarrete-Navarrete N, Peralta-Ramírez MI. Adherence to treatment in patients with systemic lupus erythematosus. Med Clin(Barc) 2018; 150(1):8-15. doi: 10.1016/j.medcli.2017.05.035.

12. Flower C, Hambleton I, Campbell M. The Effect of Psychosocial and Neuropsychiatric Factors on Medication Adherence in a Cohort of Women With Systemic Lupus Erythematosus. J Clin Rheumatol 2016; 22(8):411-17. doi:10.1097/RHU. 0000000000000470.

13. Yilmaz-Oner S, Oner C, Dogukan FM, Moses TF, Demir $\mathrm{K}$, Tekayev N. et al. Health-related quality of life assessed by LupusQoL questionnaire and SF-36 in Turkish patients with systemic lupus erythematosus. Clin Rheumatol 2016; 35(3):617-22. doi: 10.1007/s10067-015-2930-1.

14. Rodríguez Huerta MD, Trujillo-Martín MM, RúaFigueroa Í, Cuellar-Pompa L, Quirós-López R, SerranoAguilar P. Healthy lifestyle habits for patients with systemic lupus erythematosus: A systemic review. Semin Arthritis Rheum 2016; 45(4):463-70. doi: 10.1016/j.semarthrit.2015.09.003.

15. Akbarian M, Faezi ST, Gharibdoost F, Shahram F, Nadji A, Jamshidi AR. et al. Systemic lupus erythematosus in Iran: a study of 2280 patients over 33 years. Int J Rheum Dis 2010； 13(4):374-79. DOI: $10.1111 /$ j.1756185X.2010.01547.x. 
16. Akbarian M, Faezi ST, Gharibdoost F, Shahram F, Nadji A, Jamshidi AR. et al. The epidemiology of systemic lupus erythematosus in Iran: a survey on 2143 cases. Tehran Univ Med J 2010; 68(5).

17. Dabaghian FH, Rassouli M, Sadighi J, Ghods R. Adherence to prescribed medications of Iranian traditional medicine in a group of patients with chronic disease. $\boldsymbol{J}$ Res Pharm Pract 2016; 5(1):52-57. doi: 10.4103/2279042X.176563.

18. Hosseinalipour SA, Mohammadi M, Rahbar A, Mohammadbeigi A, Arabshahi A, Mohebi S. Assessment of Adherence to Anti-Tuberculosis Treatment among Patients with Tuberculosis in Qom Province in 2019 (Iran). Qom Univ Med Sci J 2020; 14(1):1-10. doi.org/10.29252/qums.14.1.1.

19. Prudente LR, de Souza Diniz J, Ferreira TXAM, Lima DM, Silva NA, Saraiva G. et al. Medication adherence in patients in treatment for rheumatoid arthritis and systemic lupus erythematosus in a university hospital in Brazil. Patient Prefer Adherence 2016; 10:863-70. doi: 10.2147/PPA.S79451. eCollection 2016.

20. Nossent J, Kiss E, Rozman B, Pokorny G, Vlachoyiannopoulos P, Olesinska $M$. et al. Disease activity and damage accrual during the early disease course in a multinational inception cohort of patients with systemic lupus erythematosus. Lupus 2010; 19(8):949-56. doi: $10.1177 / 0961203310366572$.
21. Mehat P, Atiquzzaman M, Esdaile JM, AviÑa-Zubieta A, De Vera MA. Medication Nonadherence in Systemic Lupus Erythematosus: A Systematic Review. Arthritis Care Res (Hoboken) 2017; 69(11):1706-13. doi: 10.1002/acr.23191.

22. Amalia R, Sasongko H, Kundarto W, Niruri R. The Analysis of the Factors affecting Medication Adherence in Patient with SLE (Systemic Lupus Erythematosus) at Yayasan Tittari Griya Kupu Solo. Indian J Pub Health Res Dev 2019; 10(3):672-76. doi.org/10.5958/09765506.2019.00580.1.

23. Chehab G, Sauer GM, Richter JG, Brinks R, Willers R, Fischer-Betz R. et al. Medical adherence in patients with systemic lupus erythematosus in Germany: predictors and reasons for non-adherence - a cross-sectional analysis of the LuLa-cohort. Lupus 2018; 27(10):1652-60. doi: $10.1177 / 0961203318785245$.

24. van den Bemt BJF, Zwikker HE, van den Ende CHM. Medication adherence in patients with rheumatoid arthritis: a critical appraisal of the existing literature. Expert Rev Clin Immunol 2012; 8(4):337-51. doi: 10.1586/eci.12.23.

25. Mahmood S, Jalal Z, Hadi MA, Orooj H, Shah KU. NonAdherence to Prescribed Antihypertensives in Primary, Secondary and Tertiary Healthcare Settings in Islamabad, Pakistan: A Cross-Sectional Study. Patient Prefer Adherence 2020; 14:73-85. doi: 10.2147/PPA.S235517. 